\title{
Wavelet based Reversible Image Watermarking with Logistic Encryption for Health Informatics Systems
}

\author{
B. Vijayakumar, Smita Khond
}

\begin{abstract}
Health Informatics systems preserves the patient's digital records. Two techniques that help in this process are watermarking and encryption. In this paper a reversible image watermarking scheme with logistic encryption is presented. The reversible watermarking is utilizing the concept of integer wavelet transform. The image is divided into sub bands and then the binary data is hidden in these sub bands. The watermarked wavelet sub bands are passed through logistic encryption module which scrambles the coefficients. These coefficients are then sent to inverse wavelet transform for image reconstruction. This process helps encrypt the image though spectral scrambling, thus resulting in faster and better encryption. The proposed algorithm outperforms the exiting algorithms in terms of execution time and the level of encryption.
\end{abstract}

Index Terms: reversible data hiding, encryption, wavelet, sub bands.

\section{INTRODUCTION}

In remedial uses as well as in the enhancement of investigative abilities, Medical image analysis is broadly utilized to assist Doctors. Confidentiality complication such as, alteration and unapproved admission might be initiated by means of the Broadcast of the remedial data over a communal web. Numerous explores have been implemented in order to report these complications and conveyed results on behalf of content validation in this esteem. Remedial image watermarking sources unique image adaptation and alteration even though digital image watermarking has marked the afore said complications [1].The exclusive image matter needs to be conserved because even a slight alteration in medical image watermarking ought to undesirable influence on physician investigative [2]. Towards restoring both the exclusive image as well as watermark data adjustable watermarking has stood presented by means of an authentic manner in latest centuries. Patient confidentiality is reserved as well as the physician investigative procedure and conducts remain unaffected using this method. By means of a significant portion of Health Information System (HIS), Reversible image watermarking can remain deliberated. In the investigation communal, reversible watermarking has appealed a percentage of responsiveness in recent times. Towards attaining improved strength, watermarking by means of transform domain which is attentive on the wavelet transform [3],is employed in numerous latest revisions.
Intended for reversible watermarking, Haar discrete wavelet convert remained utilized. In Haar distinct wavelet range, transformation enlargement grounded reversible watermarking is employed. In distinct wavelet transmute Kumar et al. hired unusual assessment disintegration centered reversible watermarking. By means of a quantization service, fourth stage of distinct wavelet convert was employed on behalf of implanting. En route for attainment of additional safety, Watermark statistics was preset using BCH coding [4].Towards producing a distorted image, Selvam et al. initially employed numeral wavelet convert in addition to that they practiced distinct Gould transform in distorted factors then extended a capability of 0.25 for each pixel. Numeral wavelet convert was employed, by Cohen- Daubechies Fauraue. The histogram was precontrolled towards avoiding the excess or inflow besides at that moment further data was produced [5]. For hardiness and softness of the distorted factors, Companding was practiced. Mean while here is a possibility of alteration in companding progression as a result further data was produced. Using additional alteration as well as methods, the stimulating of the reversible watermarking has been explored using specific of the preceding revisions. Through changing the mean assessment of factors, Authors of estimate Slantlet convert of a portion as well a simplant the watermark bit. Watermarking was distorted near Arnold purview and excess or inflow remained stopped using post-dispensation towards improving the safety. A section of concern and non-section of concern remained detached inevitably at leading by means of adaptive threshold indicator procedure[6]. Formerly, by means of bin histogram individually, inserting in every extent was employed. Centered on estimate fault extension, reversible image watermarking was anticipated. When excess or inflow arose transverse neighbors were assumed by means of implanting situations. Adjustably histogram fluctuating was accomplished openly to pixels or else estimate faults. On behalf of watermarking usingexclamationfaultextension2 intellectual practices comprising "genetic algorithm" and "particle swarm optimization" were employed [7-8].Aimed at damage recognition, intermediary substantial bit replacement was employed by means of watermark implanting procedure, as well as a insubstantial watermark was implanted. Towards increasing the safety, Watermark bits were encoded earlier to implanting. 
In instance of encoded images, Reversible watermarking can be advantageous. Therefore on behalf of encoded images, a contingency matter of Paillier cryptosystem was employed [9-10].Similarly, for the encoded image portion histogram changing was employed. A unique reversible watermarking scheme for remedial images on teleremedy uses is anticipated in this paper. In 2 repetitions, Numeral wavelet purview is employed on behalf of watermarking in addition to that watermark bits are implanted in every sub-group. Factors are improved using implanting in the leading repetition. Factors are improved in such a fashion that to be adjacent to the unique standards in the succeeding repetition. The aforementioned is likely to need two-bit implanting using minor alterations in this way. Likewise, constant alteration in the leading repetition is recompensed in the succeeding repetition.

\section{REVERSIBLE DATA HIDING TECHNIQUE WITH LOGISTIC ENCRYPTION}

By means of a convert purview grounded reversible image watermarking, the distinct wavelet convert is expansively employed in latest revisions. Pixels remain transformed in the custom of numeral standards towards the variable topic 1 in distinct wavelet convert [11]. Conservation of unusual numeral significance cannot be assured owed towards altering the constant standards in implanting stage in the custom of shortening. In the anticipated reversible image watermarking, number to number wavelet convert is engaged towards reporting this difficulty. Twofold stages comprising implanting plus abstraction remain convoluted in the anticipated process [10].Wavelet constants stay transformed into a dualistic plot in the anticipated procedure using:

$$
Q(x)=\bmod \left(\left[\frac{x}{2}\right], 2\right)
$$

Here, aimed at computing the remains, "mod" is a value in addition[.] is a ground value. The unusual constant assessment remains transformed by means of a continual level affording towards the waterline bit as well as equivalent dualistic plot. In the course of the restoration of the unusual image, this method may well generate the identical level aimed at dissimilar constants as well as origins uncertainty. Follower fundamental is formed as adjacent data and marks the procedure adjustable towards avoiding this uncertainty. Implanting as well as abstraction stages remain offered in further particulars in the foregoing.

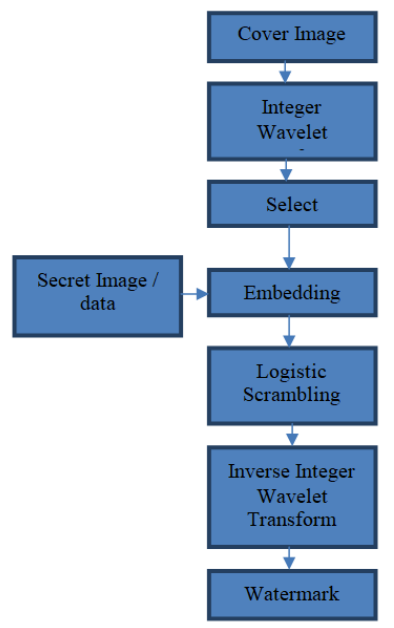

Fig 1. Proposed Algorithm

\section{EMBEDDING STEPS}

\section{A. Embedding phase}

In Fig. 1 a summary of the proposed algorithm is presented. Cover image and a waterline symbol are the structure contributions and watermarked image and tracing fundamental remain the structure productivities. Comprising LL, LH, HL, plus HH, the shield image is distorted by means of single stage numeral wavelet convert, as well as 4 wavelet sub-groups are anticipated mainly. Implanting in undersized-frequency sub-group and consuming great considerate towards the human optical structure, LL sub-group can be directed towards noncognitive alteration. Therefore, 3 sub-groups of the extreme-frequency (LH, HL, and $\mathrm{HH}$ ) remain elected on behalf of implanting affording towards proficiency necessities. Aimed at every particular implanting sub-group, the amount of waterline moments meant for implanting remains distributed keen on twofold equivalent portions. Implanting stage consists of twofold repetitions, besides every single moment of leading portion remains implanted on a factor in the leading repetition. Succeeding portion of the waterline remains implanted in the formerly implanted factors in the succeeding repetition.

\section{Algorithm 1}

1. Select the sub-band for Embedding.

2. Initialize the counter of embedded watermark in diagonal subband

3. Iterate till all the data bits are embedded

3.1. Extract watermark bits

3.2. For every value in the subband matrix

3.2.1. Find if the number is even or odd.

3.3. For the first half of the embedding data bits

3.3.1. If the value is odd and the watermark value is 0 Increment the value by 2

3.3.2. If the value is even, and the watermark value is 1 Increment the value by 2

3.4. For the second half of the embedding data bits

3.4.1. If the value is even and the watermark value is 0 Decrement the value by 2

3.4.2. If the value is odd, and the watermark value is 1

Decrement the value by 2

In Algorithm1, Implanting procedure aimed at twofold repetitions is described. Through converse numeral wavelet convert, the implanted image in wavelet area remains distorted posterior to the spatial field. Through significance slighter than zero as well as excess aimed at individuals through a assets superior than 255Implanting procedure might indicates to a charge external of the adequate collection of the image standards that remain in flow aimed at pixels. Pixel standards remain condensed as well as their positions and their unique standards stand deliberated by means of adjacent data for these circumstances. Assume that wavelet constant remains $(u, v)$,as well as the dimension of the waterline by means of adjacent data is $(i) i=1, \ldots$,and waterline remains $w$ as well as waterline d constant remains $c w(\mathrm{u}, \mathrm{v})$. Implanting procedure (Algorithm1) is given below. Dissimilarities of repetition 1 are recompensed in such a manner that 
Waterline data is implanted using repetition 2. The three advantages of the Implanting procedure are specified as follows. In the leading method, amount of improved constants remains insignificant which points towards small noncognitive perversion. Furthermore in the succeeding method, dissimilarities organized factors commencing repetition 1 can remain recompensed in repetition 2 . Using the capability of implanting in every single sub-band ( $\mathrm{LH}$, $\mathrm{HL}, \mathrm{HH})$, the situation remains likely to surge the implanting capability in the next method. Therefore, we have attained extreme volume of 1.5 BPP. Extra sturdiness can be attained using modest approaches such as reproducing in addition to this, the aforementioned is a significance declaration.

\section{B. Logistic Scrambling}

$$
x_{n+1}=\lambda x_{n}\left(1-x_{n 2}\right)
$$

Value of $\mathrm{x}$ at $\mathrm{n}$ plus 1 time is determined by value of $\mathrm{x}$ at the inner time and this parameter called lambda. In other words you start with some starting value of $\mathrm{x}$ called $\mathrm{x} 0$ and it is simply iterated over different time steps to get $\mathrm{x} 1$. The same process continuous to get the next values in the equation. Lambda plays a major role in this equation. If the value of lambda is less than 3 , the result started from 0 and eventually after say 20 or so iterations it reached a fixed point. For lambda between 3 and 3.5, X starts oscillating between two points, in this case 0.8 and 0.5

When lambda value is increased beyond 3.5, a chaotic pattern is produced as shown in figure below.

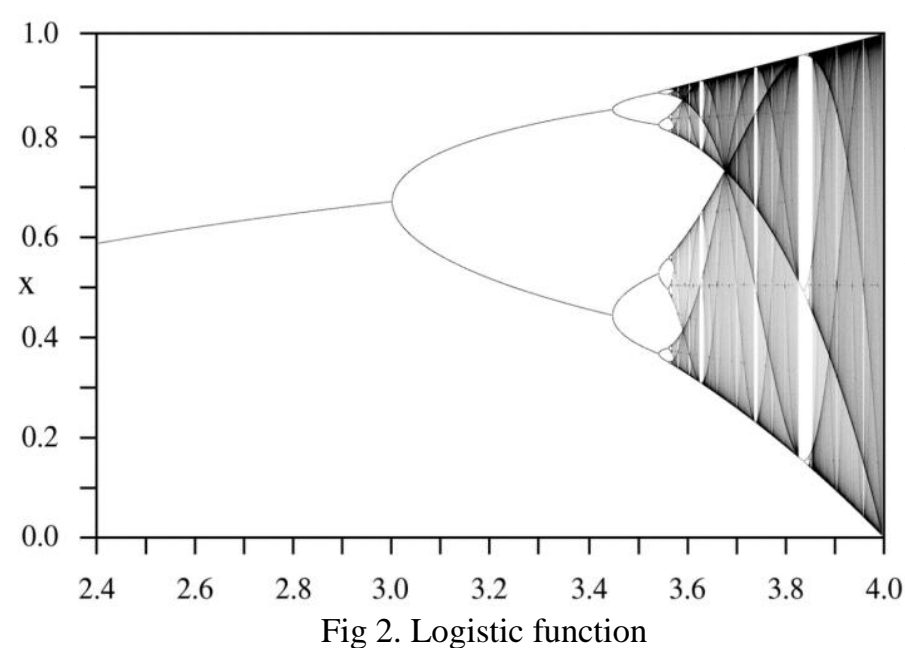

On the $\mathrm{x}$ axis - values of lambda

On the $\mathrm{y}$ axis - values of $\mathrm{X}$

If iterated a long time, so for example like for $\mathrm{X}$ less than 3 , it always $X$ for lambda. $X$ less than 3 , it always finds a single stable point for lambda between 3.1 and about 3.5 it oscillates between two points and then X starts oscillating between four points and then very quickly for eight points and even further $\mathrm{X}$ is oscillating between 16 points and after that it's just chaos it's completely unpredictable and the value that $\mathrm{X}$ takes is actually used in a lot of pseudo-random number generators because it is unpredictable.

Improved logistic map:

$x_{\mathrm{n}+1}=r * x *(1-x) * g-$ floor $(r * x *(1-x) * g)$

(3)

$$
\left.x_{n+1}=\bmod \left(1000 *\left(\left(x_{n+1}+a b s\left(x_{n+1}\right)\right)\right)\right) \cdot 1\right)
$$

This chaotic behavior is used to scramble the images in image processing domain. In this paper the logistic scrambling based encryption is applied in wavelet domain at the time of data hiding. This reduces the time of execution when compared to the traditional techniques.

\section{Extraction phase}

In Fig. 2, general ideas of the anticipated abstraction stage remain obtainable. Watermarked image and tracing fundamental are the Participations in addition to it, enhanced unusual image and detached waterline logos remains the productivities. The adjacent data stands utilized to improve the excess/inflow standards rear to their unusual positions on top, by means of a pre-succeeding period. Spatial-purview forms of the watermarked image are recovered afterwards. Numeral wavelet convert is accomplished on watermarked image in addition to it, 4 sub-groups are acquired afterwards the pre-succeeding. In twofold repetitions through recompense procedure, Watermark data is detached after implanted sub-groups. Single moment remains detached commencing every wavelet factor on every single repetition. The reversed directive of the implanted remains the directive on which watermark data is detached. Commencing leading repetition, implanted watermark in the succeeding repetition is detached. Using reverse numeral wavelet convert, the unusual image is restored as a final point. For abstraction stage, the constant rate is $(u, v)$, in addition to dimension of watermark as adjacent data remainst $(i) i=1, \ldots$, and detached watermark is we,and improved factor iscr $(u, v)$. The procedure of extraction (Algorithm2) of the anticipated watermarking technique is given below.

\section{Algorithm II.}

1. Select the sub-band for Embedding.

2. Initialize the counter of embedded watermark in diagonal sub band

3. Iterate till all the data bits are embedded

3.1. Extract watermark bits

3.2. For every value in the sub band matrix

3.3. Perform modulo operation on the value to extract the watermark

3.4. For the first half the embedding data bits

3.4.1. If the bit is 0 .

\subsubsection{If the bit is 1}

Increment the value by 2

Increment the value by 2

3.5. For the second half the embedding data bits

3.5.1. If the bit is 0 .

Increment the value by 2

3.5.2. If the bit is 1

Increment the value by 2

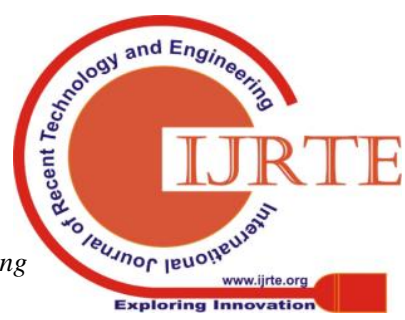




\section{RESULTS}

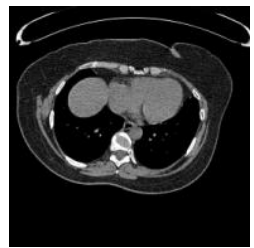

(a)

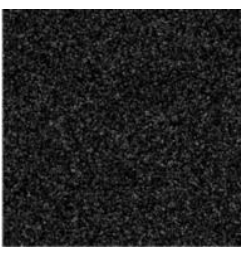

(a)

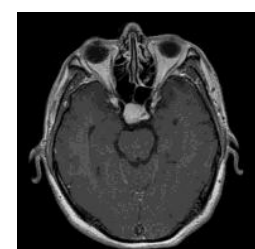

(b)

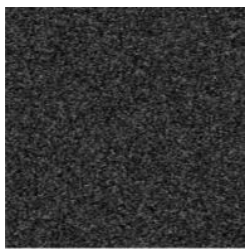

(b)
Fig.3. Input Images

Fig.4. Watermark Images

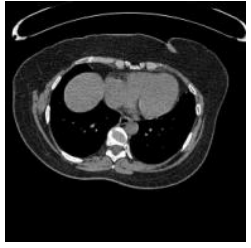

(a)

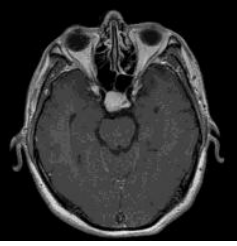

(b)

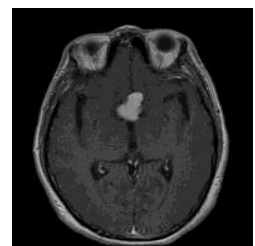

(c)

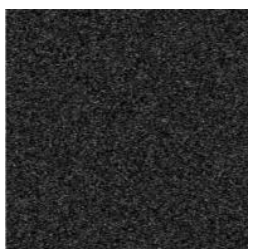

(c)

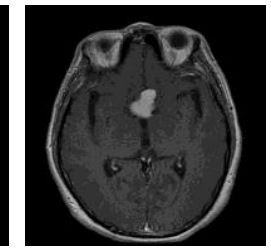

(c)
Fig.5. Output Images

The correlation index (Corr2) measures the similarity of the input images with the output encrypted images. The table 1 represents the correlation index of the images considered for experiments. The proposed algorithm provides better encryption compared to the existing algorithms.

Table 1: Correlation Values

\begin{tabular}{|c|c|c|c|c|c|}
\hline $\begin{array}{c}\text { S. } \\
\text { No. }\end{array}$ & Image & SHA256 & $\begin{array}{c}\text { Chaotic } \\
\text { encryptio } \\
\mathrm{n}\end{array}$ & $\begin{array}{c}\text { DNA } \\
\text { Encryption }\end{array}$ & $\begin{array}{c}\text { Proposed } \\
\text { Method }\end{array}$ \\
\hline 1 & $3(\mathrm{a})$ & 0.0013 & 0.0029 & 0.000435 & -0.0059 \\
\hline 2 & $3(\mathrm{~b})$ & 0.004 & 0.0038 & 0.0010 & $\begin{array}{c}-0.00097 \\
7\end{array}$ \\
\hline 3 & $3(\mathrm{c})$ & 0.0029 & 0.003 & 0.000869 & -0.0039 \\
\hline
\end{tabular}

Table 2: Execution time

\begin{tabular}{|l|l|l|}
\hline $\begin{array}{l}\text { S. } \\
\text { No. }\end{array}$ & Image & $\begin{array}{l}\text { Proposed } \\
\text { Algorithm }\end{array}$ \\
\hline 1 & $3(\mathrm{a})$ & $1.35 \mathrm{~s}$ \\
\hline 2 & $3(\mathrm{~b})$ & $1.62 \mathrm{~s}$ \\
\hline 3 & $3(\mathrm{c})$ & $1.60 \mathrm{~s}$ \\
\hline
\end{tabular}

\section{CONCLUSION}

Novel reversible image watermarking method with logistic encryption was presented for medical images based on integer wavelet transform. Improving distortion and embedding capacity was considered in the proposed method. The embedding process was performed in two iterations using a compensation approach. It was possible for modified coefficients in the first iteration, to recover its original value in the second iteration. One bit was embedded on each iteration. Hence the maximum capacity of $1.5 \mathrm{BPP}$ was obtained. Simulation results demonstrated that the proposed reversible image watermarking provided suitable capacity-distortion in comparison with the other methods.

\section{REFERENCES}

[1]A. Draganić, M. Marić, I. Orović, S. Stanković, "Identification of image source using Serial-number-based watermarking under Compressive Sensing conditions," 40 th International Convention on Information and Communication Technology, Electronics and Microelectronics, MIPRO 2017

[2] Rai A, Singh HV (2017) SVM based robust watermarking for enhanced medical image security. Multimed Tools Appl, 1-14.

[3] J. Huang, G. Chen, L. Shu, S. Wang, and Y. Zhang, "An experimental study of clogging fault diagnosis in heat exchangers based on vibration signals,' IEEE Access, vol. 4, pp. 1800-1809, Jun. 2016.

[4] Soleymani SH, Taherinia AH (2016) Double expanding robust image watermarking based on spread spectrum technique and $\mathrm{BCH}$ coding. Multimedia Tools Appl 12:1-9.

[5] Tan MH, Li Q, Shanmugam R, Piskol R, Kohler J, Young AN, et al Dynamic landscape and regulation of RNA editing in mammals. Nature. 2017;550:249-54.

[6] Tran, L. An interactive method to select a set of sustainable urban development indicators. Ecol. Indic. 2016, 61, 418-427.

[7] E. Ruijters, S. Schivo, M. Stoelinga, and A. Rensink, "Uniform analysis of fault trees through model transformations," in 2017 Annual Reliability and Maintainability Symposium (RAMS), Jan 2017, pp. 1-7.

[8] Yuan, X., Elhoseny, M., Minir, H., Riad, A., 2017. A genetic algorithm-based, dynamic clustering method towards improved WSN longevity. Journal of Network and Systems Management, Springer US 25 (1), 21-46. http://dx.doi.org/10.1007/ s10922-016-9379-7.

[9] Wu, H.-T.; Cheung, Y.-M.; Huang, J.: Reversible data hiding in Paillier cryptosystem. J. Vis. Commun. Image Represent. 40, 765- 771 (2016).

[10] Han, H., Lee, M.J., \& Hwang, J. (2016). Cruise travelers' environmentally responsible decision-making: An integrative framework of goal-directed behavior and norm activation process. International Journal of Hospitality Management, 53, 94-105.

[11] Hamidreza Zarrabi, Mohsen Hajabdollahi, S.M.Reza Soroushmehr,Nader Karimi, Shadrokh Samavi, Kayvan Najarian, "Reversible Image Watermarking for Health Informatics Systems Using Distortion Compensation in Wavelet Domain", 2018 40th Annual International Conference of the IEEE Engineering in Medicine and Biology Society (EMBC), July 2018.

\section{AUTHORS PROFILE}

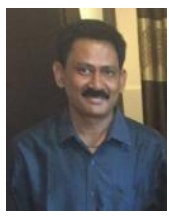

Dr.B. Vijayakumar is Professor in Computer Science \& Engineering in Vidya Jyothi Institute of Technology (VJIT) ,Hyderabad. He has about 23 years of Academic and Industry experience. $\mathrm{He}$ is a Life Member of CSI,ISTE,NESA,ISCA. He has more than 50 publications in the field of Image Processing, Digital Watermarking, IoT and Cloud Computing.

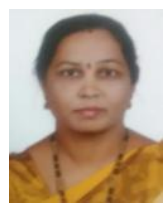

Smita Khond is a research scholar under the guidance of Dr B.Vijayakumar from Pacific University (PAHER) Udaipur,Rajasthan. She is working as Associate Professor in Computer Science and Engineering (CSE) Department of Malla Reddy Engineering College for Women ,Hyderabad 